\section{$\underset{\substack{\text { hommes } \\ \text { \& migrations }}}{ }$}

\section{Hommes \& migrations}

Revue française de référence sur les dynamiques

migratoires

$1298 \mid 2012$

France - Algérie, le temps du renouveau

\title{
Entre exils, errances et migrations
}

L'expérience littéraire de Kateb Yacine

\section{Kaoutar Harchi}

\section{(C) OpenEdition}

1 Journals

\section{Édition électronique}

URL : http://journals.openedition.org/hommesmigrations/1584

DOI : 10.4000/hommesmigrations. 1584

ISSN : 2262-3353

Éditeur

Musée national de l'histoire de l'immigration

\section{Édition imprimée}

Date de publication : 1 juillet 2012

Pagination : 112-122

ISSN : 1142-852X

\section{Référence électronique}

Kaoutar Harchi, «Entre exils, errances et migrations », Hommes \& migrations [En ligne], 1298 | 2012,

mis en ligne le 01 juillet 2014, consulté le 19 avril 2019. URL : http://journals.openedition.org/

hommesmigrations/1584; DOI : 10.4000/hommesmigrations.1584 


\section{Entre exils, errances et migrations}

L’expérience littéraire de Kateb Yacine

Par Kaoutar Harchi, doctorante-allocataire-monitrice en sociologie, Paris, Sorbonne-Nouvelle, CERLIS

La vie de l'écrivain algérien Kateb Yacine s'est nourrie de voyages qui ont modelé son écriture. L'emploi de la langue française est le reflet d'une identité troublée qui cherche à refonder ses lieux, ses liens, son langage par-delà la domination étrangère. Dans Nedjma où le légendaire côtoie le réel, son écriture polyphonique fait tourbillonner les blessures de l'histoire et les traces d'une mémoire à retrouver. Si elle catalyse les souffrances d'un peuple, l'œuvre de Kateb Yacine traduit avant tout les dynamiques profondes d'une identité migrante. 
Fontaine de sang, de lait, de larmes, elle savait d'instinct, elle, comment ils étaient nés, comment ils étaient tombés sur la terre, et comment ils retomberaient, venus à la brutale conscience, sans parachute, éclatés comme des bombes, brûlés l'un contre l'autre, refroidis dans la cendre du bûcher natal, sans flamme ni chaleur, expatriés. Kateb Yacine, Le Polygone étoilé(1)

Nedjma a été, et cela dès sa publication, placé sous d'étranges augures qui pouvaient laisser présager le destin singulier que ce roman de langue française, ainsi que son auteur algérien, Kateb Yacine, connaîtraient. Dans leur "Avertissement", "Les Éditeurs" mettaient les lecteurs en garde contre ce qui, à leurs yeux, était "une cuvre profondément arabe et sur laquelle on ne peut porter un jugement valable si on la sépare de la tradition à laquelle, jusque dans ses reniements, elle ne cesse d'appartenir ${ }^{(2) ", ~ e t ~ i l s ~}$ ajoutaient : "La pensée européenne se meut dans une durée linéaire, la pensée arabe évolue dans une durée circulaire où chaque détour est un retour, confondant l'avenir et le passé dans l'éternité de l'instant ${ }^{(3)}$." Manière étonnante que d'inscrire, avec autant d'assurance, ce roman dans une culture, une civilisation précise et définitive, sans lui accorder le droit d'être d'ailleurs, de plusieurs lieux à la fois, ou même de nulle part. Ce droit à l'hétérodoxie, nous pensons que Kateb Yacine, chaque jour de sa vie, se l'est pourtant octroyé, pour son travail comme pour lui-même.

L'homme a voyagé, de lieux prévisibles en lieux imprévus, convaincu qu' il n'y a plus alors d'Orient ni d'Occident', recherchant partout, pour sa pensée, de nouveaux espaces à investir, où se renouveler. Pour Nedjma, sa cousine-muse jamais possédée. Et contre la violence inédite des archaïsmes, la folle fureur coloniale, la fatalité de la faim, "le vent qui ne veut jamais rester dehors"(4)". L'écriture est liée à ces vagabondages. Elle leur a donné sens et forme. Les a fixés afin de permettre le souvenir autant que la complainte. C'est ainsi peut-être que nous pouvons reconnaître, derrière ces propos de Lakhdar, personnage central du Polygone étoilé, l'expérience du racisme, réellement vécu par Kateb Yacine, en 1955, lorsqu'il devient docker sur le port de Marseille: "Faut des papiers prouvant qu'on a déjà travaillé, faut passer tous les jours, ils ont pris l'habitude de voir des Algériens à leur porte. C'est loin. À notre époque, un chômeur consciencieux devrait avoir une bicyclette, et des habits qui ne les dégoûtent pas. Il devrait les écouter en défilant éternellement devant leurs bureaux, en cachant ses cheveux frisés, le nombre de ses enfants et de ses maladies puisqu'ils ont peur de payer trop de cotisations ${ }^{(5)}$."

À travers ces quelques lignes transparaît l'imbrication forte qui unit l'expérience intime du mépris manifeste d'une partie de la société française à l'égard des 
travailleurs immigrés et sa nécessaire reconfiguration esthétique. Expérience intime qui, ayant la particularité d'être vécue collectivement (le "on" impersonnel prend ici toute son importante), fait disparaître la confession, au profit du déploiement d'un puissant lamento.

Quels liens existent-ils entre le déplacement et la création dans la pratique littéraire de Kateb Yacine? Quelle(s) logique(s) sous-tende(nt) ses exils compulsifs survenus avant et après la guerre d'Algérie? De quelles manières ces expériences rejaillissentelles sur sa création? Afin de répondre à ces interrogations, nous explorerons trois pistes de recherche. Dans un premier temps, nous reconstruirons le parcours biographique de l'auteur. Dans un second temps, nous nous intéresserons au motif de la mobilité dans l'ceuvre romanesque katébienne. Dans un troisième temps, nous nous attacherons à reconstruire les stratégies d'écriture développées par l'écrivain. Ces axes de recherche incluront, autant que possible, des éléments de comparaison précisant, notamment, des éléments relatifs à la pratique littéraire de l'écrivaine algérienne Assia Djebar et de l'écrivain algérien Mohammed Dib, cela afin que l'on puisse saisir les singularités et les régularités propres à la trajectoire migratoire de Kateb Yacine.

\section{Une existence vouée à l'Ailleurs}

Année : 1929. Kateb Yacine serait vraisemblablement né le 6 août. Lieu : Constantine. Ville ô combien importante si l'on se souvient de la place qu'elle occupe dans le roman Nedjma et qui est, "conjointement avec Bône ${ }^{(6)}$ ", "une cité d'attente et de menace ${ }^{(7) " . ~ L ' e s p a c e, ~}$ tel que décrit par l'auteur, est le lieu d'un conflit historique qui fragilise depuis 1830 l'équilibre de la société kabyle. À cela s'ajoutent des difficultés internes à cette même société. La faiblesse de la production agricole contraint l'individu à rechercher constamment la protection de son groupe d'appartenance. Comme l'écrit Pierre Bourdieu, "les caractères sociologiques des populations algériennes forment un écheveau inextricable ${ }^{(8)}$,' et la tribu se révèle être un puissant foyer de cohésion et de solidarité. Kateb Yacine précise: "Mon père était avocat, mon père et ma mère, mon grand-père et ma grand-mère, mes oncles, mes tantes, tout ça,ça vient de la même tribu, et nous sommes tous issus de mariages consanguins ${ }^{(9)}$."

Ce père, oukil, a très souvent été muté et la famille entière l'a suivi dans ses déplacements professionnels. Jusqu'à l'âge de 8 ans, le jeune Yacine a vécu à Sédrata, ville rendue douloureuse par les drames familiaux, comme le suggère ce passage : "J'écris ces lignes à Sédrata, sur la tombe de mon frère aîné Belghith, mort de nostalgie à l'âge de deux ans ${ }^{(10)}$." Cette période de la vie de Kateb Yacine ne peut être reconstituée qu'à partir de très minces souvenirs d'enfance, dont la cohérence et la logique 
viennent parfois à faire défaut tant la fixation mnésique et la charge affective qui s'y rattachent semblent les avoir travaillés et déformés. Notons simplement qu'après Sédrata, il y eut Bougâa, puis Guelma et, trente-cinq kilomètres plus loin, Souk Ahras, puis à six heures de route, Sétif.

L'apprentissage de la langue française, au cours de ses études secondaires, provoque en Kateb Yacine des déchirements qui l'entraînent toujours plus loin dans le sentiment d'abandonner sa langue maternelle ainsi que sa propre mère. Il précisera : "Jamais je n'ai cessé (...) de ressentir au fond de moi cette seconde rupture du lien ombilical, cet exil intérieur (...). Ainsi avais-je perdu tout à la fois ma mère et son langage, les seuls trésors inaliénables et pourtant aliénés ${ }^{(11)}$." La question coloniale apparaît alors comme une mise en concurrence permanente d'ensembles linguistiques luttant pour imposer leur suprématie respective.

L'appréhension de l'avènement de la colonisation en tant que ruine irréversible des rap-

En 1959, expulsé
d'Algérie par les autorités
coloniales pour son
militantisme communiste,
Mohammed Dib migre
vers la France.
ports familiaux et du patrimoine linguistique traditionnel algérien n'est pas véritablement partagée par l'écrivain francophone Mohammed Dib. Né en 1920, à Tlemcen, dans l'Ouest algérien, au sein d'une famille de la petite-bourgeoisie - en plus de manier parfaitement le français, en raison de sa fréquentation de l'École française d'Alger -, Mohammed Dib s'initie à l'anglais et, s'ajoutant aux ceuvres françaises de Nathalie Sarraute et d'Alain RobbeGrillet, il découvre celles d'Arthur Miller, de Virginia Woolf, de James Joyce, appréciant le procédé du courant de conscience. Cette admiration pour "l'écriture et la langue du yankee" ne va pas sans interroger le rapport de Dib à la langue française et à sa langue maternelle, l'arabe. En 1959, expulsé d'Algérie par les autorités coloniales pour son militantisme communiste, Mohammed Dib migre vers la France. L'auteur de La Grande Maison ${ }^{(12)}$ et de L'Incendie ${ }^{(13)}$ engagera dès lors un long travail grâce auquel il parviendra à assumer, sans déchirure, le choix de la langue des Autres comme lieu privilégié de lecture, d'écriture, et il ira jusqu'à dire :"Le français est devenu ma langue adoptive (...), non pas de manière inconsciente mais comme une marche (...), une recherche de soi."

Nous mesurons là à quel point l'expérience du déplacement (à l'intérieur d'un même pays ou entre divers pays) a des répercussions intenses sur l'ensemble des domaines de l'existence humaine. La création littéraire, parce qu'elle nécessite un grand effort de réflexivité, amplifie le sentiment de bouleversement vécu par l'individu et le conduit à se positionner, socialement, de manière à pouvoir donner du sens, poétiquement, à ce qu'il a vécu. 


\section{Une rupture existentielle décisive}

Le 8 mai 1945 éclatent la révolte et les massacres de Sétif et Guelma. Kateb Yacine participe à ce soulèvement pour l'indépendance algérienne. Il est emprisonné quatre mois durant. L'expérience de la détention a profondément modifié la vision que le jeune homme avait de la présence coloniale. Pour la première fois de sa vie, il approche, voit, ressent, la souffrance de son peuple. S'affirment là son identification et son appartenance viscérale à la nation algérienne. Pourtant, à partir de 1947, la présence de l'auteur en Algérie ne se fera plus que par intermittence. Ses séjours à Paris, eux, se multiplient. En 1949, mandaté par le journal Alger républicain, Kateb Yacine se rend à Djeddah, en Arabie Saoudite. Puis ce sera le Soudan, en 1950, l'Union soviétique, l'Ouzbékistan, la France, Marseille et son port, Paris à nouveau, la Tunisie, l'Allemagne, l'Italie, la Belgique. Il est difficile de préciser les raisons exactes de ces voyages et leur durée, néanmoins nous pouvons affirmer qu'à travers ce vagabondage, se met en place une pratique littéraire caractérisée par le récit d'aventures, le carnet de voyages, l'article succinct. Documents tantôt publiés dans les journaux et revues de l'époque, tantôt égarés, définitivement ; ce qui participe à conférer à l'œuvre katébienne ce caractère fragmentaire, morcelé, brouillé, à l'image des multiples migrations et déplacements - en France, en Algérie et dans le reste du monde - de l'auteur.

En novembre 1954, lorsque la guerre éclate, Kateb Yacine est en France. Catherine Milkovitch-Rioux précise qu'à partir de là, "son instabilité s'aggravee (14)". Le jeune homme culpabilise de ne pas être là-bas, près des siens, ceux auprès desquels il aimerait lutter. Bien des années plus tard, revenant sur cette période de sa vie dans Le Polygone étoilé, il écrira : "Ceux qui ne combattent pas sont morts, sont prisonniers, s'exilent, sont bannis. (...). Beaucoup sont envahis par la nostalgie. Mais les vivres ne tarderont pas à s'améliorer, grâce à la chasse, et l'installation de la radio dans les cabanes sera déjà comme un fait d'armes, pour le moral de l'organisation. En attendant, c'est la guerre ${ }^{(15)}$.'

Kateb Yacine n'est pas le seul que cette époque va conduire à s'interroger sur le sens de la colonisation et de la libération. Dans les mêmes années, Assia Djebar, écrivaine algérienne francophone née à Cherchell en 1936, se donne pour objet de réinvestir le passé, comme une nécessité. Son inscription, en 1954, au lycée Fénelon à Paris, ses voyages fréquents au Maroc, son exil politique aux États-Unis, la confortent dans son projet d'écriture de l'Histoire, qu'elle fonde sur l'idée que "la continuité historique ne doit pas être rompue, et que l'apport de l'étranger a aussi servi à la constitution d'un devenir en gestation ${ }^{(16)}$ ". Kateb Yacine, au contraire, a été soucieux de rompre, par la littérature, avec le legs culturel des vainqueurs afin que le peuple retrouve son patrimoine artistique originel. 
Une fois l'indépendance proclamée, Kateb Yacine retourne en Algérie, mais, comme Assia Djebar et Mohammed Dib, il n'y demeure que très peu de temps. Ses vagabondages recommencent. Le nord de l'Algérie puis le Caucase, la Russie, la Chine au milieu des années soixante et, plus tard, le Vietnam. Celui qui s'appelle lui-même "l'écrivain errant" rencontre sur son chemin de futurs grands romanciers algériens, parmi lesquels nous pouvons retenir les noms de Malek Haddad, Mouloud Mammeri, Mohammed Dib lui-même. Le temps d'une journée, ils se retrouvent dans un bar, un hall d'hôtel, un quai de gare. En 1963, Kateb Yacine se confie au journaliste Philippe Bernier : "S'il ne dépendait que de moi, jaimerais beaucoup retourner vivre en Algérie, puisque c'est mon pays, parce que jaime mon pays. Mais il y a des conditions objectives qui font que je suis un écrivain errant ; ça m'a ouvert des horizons que je ne suis pas près d'abandonner. Lorsque je vais en Algérie, je reconnais franchement que je souffre, à chaque moment, parce que je suis resté trop longtemps loin du pays. Il suffit de rester absent six mois d'une ville, elle change, alors à plus forte raison si on reste absent quatorze ans ${ }^{(17) . "}$

En 1965, bien que l'Algérie soit indépendante, Kateb Yacine demeure hanté par les séquelles de la colonisation. Le recours à la langue de l'ancien colonisateur cristallise toujours plus de questionnements. C'est dans cette optique qu'il nous faudrait comprendre cet exil de nature symbolique, inédit à l'époque, dans lequel s'engage Kateb Yacine, au début des années soixante-dix : l'arrachement à la langue française pour renaître à la langue arabe dialectale.

S'adresser à son peuple, se faire comprendre de lui, tels sont les projets que se donne Kateb Yacine et desquels surgira la pièce de théâtre Mohamed prends ta valise, écrite en algérien, et jouée sur les places des marchés et dans les foyers d'immigrés. Ce besoin de revenir vers la langue maternelle est symptomatique de cette obsession que Kateb Yacine continue de nourrir à l'égard de tous ceux qui, arrachés à leur terre natale, se sont vus condamnés à l'errance et à la solitude. Soumis aux secousses de l'Histoire, le parcours de Kateb Yacine nous laisse entrevoir cette oscillation constante entre la fuite et la quête, en tant que pôles par lesquels la création romanesque, poétique et dramatique advient au monde et en ébranle durablement le sens.

\section{L'œuvre migrante}

Si l'existence de Kateb Yacine peut être perçue comme une suite de discontinuités où seule la résistance au régime colonial parvient à imposer son ordre et sa cohérence, force est de constater que son ceuvre littéraire, son roman Nedjma en particulier, porte en elle les traces de ces multiples ruptures. En ce sens, il est possible d'en dégager une fresque de l'insaisissable, une poétique du mouvement. 
Lespace référentiel tel qu'il est représenté dans Nedjma semblerait se diviser en deux catégories. D'une part, la catégorie des "espaces dramatisés ${ }^{(18)}$ ", la prison, le chantier, la rue, caractérisés par la tension, la souffrance, la mort. D'autre part, la catégorie des "espaces euphoriques", caractérisés par l'exaltation du bonheur, du plaisir, à l'instar du lieu du bain et de la campagne. Il est à noter que cette distinction entre espaces dramatisés et espaces euphoriques n'a de sens que parce qu' ils interagissent, $s$ 'influencent et finissent par se contaminer, car plus nous avançons

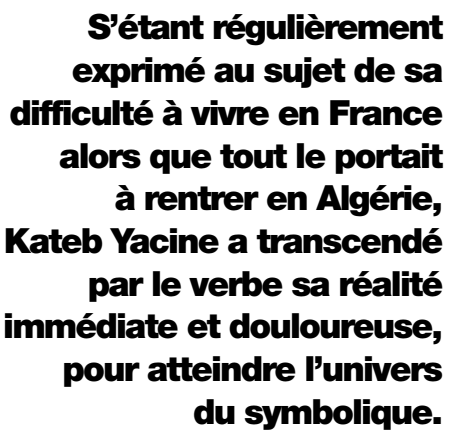

dans Nedjma, et plus le désir, autrefois pur, se confond avec la violence, se laisse submerger par elle, comme c'est le cas, par exemple, lors de la nuit de noces de Suzy et de M. Ricard, tué par Mourad.

Les lieux de Nedjma s'apparentent à une vaste mosaïque, rebelle à toute forme de saisissement définitif. Cette écriture du mouvement, de la migration se réalise "contre la fondation nationale ${ }^{(19) "}$ et tournoie autour de lignes de fuite, refusant l'avancée

directe et préférant les méandres d'une narration fondée sur le cycle. La dilatation du temps, la multiplication des scènes, l'éclatement constant du point de vue, la personnification des éléments de la Nature, tels sont les techniques qui permettent à Kateb Yacine de rompre avec les codes esthétiques du récit réaliste balzacien. Les suites de déplacements poétiques, les déambulations oniriques et tragiques à la fois, sont entièrement ouvertes et tendues vers un au-delà que ne limite aucune frontière générique. À cet égard, Catherine Milkovitch-Rioux précise qu'"au cceur de cette géométrie variable, complexe, savante, la figure migrante ne se voue pas à une expansion qui serait conquête triomphante; elle est à l'inverse l'expression de la misère vagabonde et d'une découverte errante et erratique du monde. Dans un mouvement narratif plus circulaire que linéaire, elle se voue à la répétition ${ }^{(20) "}$.

La temporalité de Nedjma fait exploser le cadre référentiel de l'époque coloniale pour remonter au moment fondateur des origines. Dans cette optique, le nomadisme forcé des hommes ne semble pas tant lié au contexte politique et économique de l'Algérie coloniale qu'à un passé mythique, portant en lui les germes d'une malédiction dont auraient à souffrir, génération après génération, tous les descendants du grand ancêtre Keblout. L'exil concerne ici non plus seulement un groupe social historique, mais bien un peuple éternel dont l'histoire serait fondée sur cette quête désespérée mais ô combien vitale d'une terre libérée de toute domination étrangère. 


\section{L'univers du symbole}

La migration s'apparente donc à une essence et non pas simplement à une condition sociale, à une existence. Nedjma reconstitue, par à-coups successifs, les aléas d'une destinée universelle regroupant en son sein tous ceux dont "le statut (...) fut toujours provisoire et chaque fois qu'on le définit, il devient un peu plus vague... (21)". La description du nomadisme se veut ralliement des plus jeunes aux plus anciens afin qu'une communauté puisse se constituer, paradoxalement, à partir du socle de son constant éparpillement.Le motif de la diaspora génère une multitude d'aventures (nous pouvons penser, par exemple, au pèlerinage à La Mecque de Si Mokhtar ou au retour au Nadhor de Nedjma) qui perturbent et reconfigurent la conception du temps et de l'espace, en les ouvrant à un champ des possibles qu'aucune tradition romanesque ne peut entièrement circonscrire car, comme l'écrit l'auteur, "les évasions ne se comptent plus ${ }^{(22)}$ ". En comparant les romans de Kateb Yacine, de Mohammed Dib et d'Assia Djebar, auteurs exilés en France à la même époque, force est de constater que Nedjma, immanquablement, se démarque, par sa facture métaphorique, de La Grande Maison et de La Soif ${ }^{233}$, plus réalistes. La situation économique et sociale dans laquelle se trouvait son auteur, Kateb Yacine, au moment de sa rédaction, était plus précaire que celle que connaissaient ses deux compatriotes, Assia Djebar et Mohammed Dib.S'étant régulièrement exprimé au sujet de sa difficulté à vivre en France alors que tout le portait à rentrer en Algérie, Kateb Yacine a transcendé par le verbe sa réalité immédiate et douloureuse, pour atteindre l'univers du symbolique. Ces éléments nous laissent croire que l'expérience de la migration, vécue par les écrivains algériens de la seconde moitié du XX' siècle, a fait l'objet d'une réintégration, plus ou moins forte mais constante, au cceur du projet littéraire lui-même. Et pour Kateb Yacine plus que pour n'importe quel autre auteur, il était question de projeter, dans l'œeuvre, les découvertes et turpitudes dues à cette impossibilité de se fixer durablement quelque part.

\section{Stratégies d’écriture, stratégies d'évasion}

Le mythe de l'ancêtre qui structure en partie Nedjma appartient à l'histoire personnelle de Kateb Yacine, lui-même véritable descendant de la tribu de Keblout. Cette transformation d'un matériau biographique en matériau littéraire ${ }^{(24)}$ fait advenir une fiction généalogique où se développe l'idée selon laquelle la fondation de tout royaume ne peut passer que par l'acceptation d'un long et périlleux exil. Comme nous le précisions déjà quelques lignes plus haut, le déplacement, s'il est une condamnation sans début ni fin, s'il s'impose à des êtres accablés, est aussi une manière 
de semer (au sens d'échapper à) le malheur, de s'affranchir de la Loi de l'Autre (ici, du colonisateur) pour fonder sa propre histoire et légende.

Le parcours de Kateb Yacine ainsi que son ceuvre littéraire nous paraissent exemplaires de cette fusion entre l'existence réelle et son prolongement imaginaire voire fantasmatique. La migration, qu'elle soit historique ou mythique, s'apparente à un processus de réinvention de soi et de son groupe d'appartenance. La migration serait ainsi dotée d'une puissante fonction de production identitaire, permettant à l'écrivain d'être non plus l'objet passif du discours, mais sa source dynamique, entraînant dans son éveil les hommes et les femmes susceptibles de se reconnaître en son verbe et en son combat. Kateb Yacine précise : "Mon activité littéraire consistait nécessairement à écrire en français et d'abord pour les Français, pour les convaincre. Il s'agissait à l'époque de montrer en français que l'Algérie n'est pas française ${ }^{(25)}$."

Bien que publiant dans une maison d'édition française, Kateb Yacine - à l'instar de Mohammed Dib et d'Assia Djebar qui revendiquent, eux aussi, un attachement profond à leur terre de naissance et dont les romans respectifs sont pensés telles des contributions littéraires au projet de libération nationale - demeure un auteur qui se revendique avant tout comme Algérien. C'est par l'appropriation, la conquête de ce "butin de guerre", c'est-à-dire la langue française, que son exil, sa migration, ses longues absences du pays natal, deviennent intelligibles, s'inscrivent dans une logique collective de reconnaissance, se chargent d'un sens précis et fédérateur, celui de la légitimité de la lutte pour la Révolution par la Poésie.

\section{L'exil, une expérience de redéfinition de soi}

La migration, vécue dans les premières années comme une forme de passage obligé pour les Nord-Africains, devient, au fur et à mesure, pour Kateb Yacine, un lieu d'expérimentation, de construction de la figure du poète engagé. La langue, l'Histoire et la politique convergent vers un objet central, point névralgique qui fonde toute action humaine, à savoir la croyance en la légitimité des peuples dominés à se libérer. L'ceuvre littéraire de Kateb Yacine a porté aux nues cet idéal en l'inscrivant dans une pluralité de vérités qui ne se voulaient pas message ou plaidoyer mais plutôt système romanesque polyphonique critiquant à la fois la raison coloniale et la raison nationaliste. Dans cette optique, écrire en français, en France, "pour expliquer aux Français que l'Algérie n'est pas française", revenait à mettre en place une puissante matrice de spécification des anciennes appartenances (à la tribu de Keblout, par 
exemple) et d'exploration des lieux primitifs d'existence (Cirta et Hippone devenues Constantine et Bône, notamment). L'impératif de régénération de l'imaginaire collectif algérien, selon Kateb Yacine, devait éliminer toute tentation d'enfermement sur soi. Pour l'écrivain, parvenir à exister politiquement signifiait parvenir à exister culturellement et artistiquement.

Assia Djebar voit, parce qu'elle est femme, son rapport à la langue française se doubler d'un niveau de complexité supplémentaire. Comme Kateb Yacine et Mohammed Dib, elle réclame le droit de s'exprimer dans la langue du colonisateur pour s'approprier ce par quoi l'entreprise coloniale avait voulu l'aliéner, mais à cela s'ajoute une volonté singulière et tenace d'offrir, grâce à la pratique scripturaire, une voix à toutes ces Algériennes affranchies par l'expérience de la guerre et que l'indépendance a tôt fait de réinscrire au cceur de l'espace domestique. Ainsi observet-on "une écriture de la transhumance", "une écriture voyageuse", "une écriture fugitive". Selon Jeanne-Marie Clerc, "cet exil imposé par le destin personnel, cette double acculturation, puis cet impossible retour au pays natal déchiré, se sont transmués en vocation d'écrivain assumant volontairement l'enracinement impossible ${ }^{(26)}$.' Si Kateb Yacine s'est résolument mis en quête des fraternités brisées par la migration, soulignons que, parallèlement à cela, l'entreprise littéraire d'Assia Djebar peut se comprendre comme une recherche des sororités tues que l'exil et la distance ont fait remonter à la surface, faisant ainsi se croiser le visible et le lisible de l'expérience cinématographique et de l'expérience littéraire.

"Milan - Tunis - Bruxelles - Hambourg - Bonn - Stockholm - Bruxelles - Milan - Monterosso - Trieste - Zagreb - Tunis - Berlin - Florence ${ }^{(27) ", ~ t e l l e s ~ s o n t ~ l e s ~ v i l l e s ~ q u ' e ́ g r e ̀ n e ~ K a t e b ~ Y a c i n e ~}$ dans le prière d'insérer du Polygone étoilé. Autant de lieux par lesquels il est passé une fois, deux fois, trois fois, seul ou avec des compagnons, sans pouvoir véritablement s'y établir, conscient que seul le passage lui était autorisé et que son chemin, plus loin encore, se prolongeait. Ce qui aurait pu s'apparenter à une errance, à une vie faite de misère et de chagrin, s'est transformé, au fur et à mesure, par l'amour de la Poésie et de la Révolution, en un destin personnel entraînant, dans son élan, tout un peuple vers une modernité artistique, socle solide de sa légitimité à vivre libre. Nedjma, avec ses zones d'ombres et ses limbes, avec ses répétitions et ses renversements de situation, décrit une Algérie à venir tout en faisant éclater le cadre supposé de ce futur avènement. Le non-lieu, le hors-champ, le mélange, le syncrétisme, le métissage, sont comme autant d'éléments indiquant le refus définitif de Kateb Yacine d'entretenir une vision monolithique de la culture et de l'art, vision dont le corollaire contemporain le plus pertinent serait certainement l'écriture du "Tout-Monde" développée par Édouard Glissant. 


\section{Bibliographie}

- Yacine Kateb, Soliloques, Bône, Imprimerie Thomas 1946 (recueil poétique); Nedjma, Paris, Seuil, 1956 (roman); Le Polygone étoilé, Paris, Seuil, 1966 (roman); L'Homme aux sandales de caoutchouc, Paris, Seuil, 1970 (théâtre); Loin de Nedjma, Alger, Laphomic, 1986 (recueil poétique).

- Abdoun Ismail, Lectures de Kateb Yacine, Alger, Casbah éditions, 2006.

- Julien Anne-Yvonne, Kateb Yacine et l'étoilement de l'ceuvre, Rennes, Presses universitaires de Rennes, 2010.

- Milkovitch-Rioux Catherine, "Figures de la migration chez Kateb Yacine et dans Nedjma".

- Bourdieu Pierre, Sociologie de l'Algérie, Paris, PUF, 1958.

- Amossy Ruth (dir.), Images de soi dans le discours. La construction de l'ethos, Paris, Delachaux, 1999.

- Albert Christine, L'Immigration dans le roman francophone contemporain, Paris, Karthala, 2005.

- Smouts Marie-Claude, La Situation postcoloniale, Paris, Presses de Sciences Po, 2007.

- Stora Benjamin, Les Immigrés algériens en France. Une histoire politique, 1912-1962, Paris, Hachette Littératures, 2009.

\section{Notes}

1. Kateb Yacine, Le Polygone étoilé, Paris, Seuil, 1966, p. 150.

2. Kateb Yacine, Nedjma, Paris, Seuil, 1956, p. 5.

3. Ibid.

4. Ibid., p. 25.

5. Ibid., p. 29.

6. Gérard Faure, "Un écrivain entre deux cultures : Kateb Yacine", in Revue de l'Occident musulman et de la Méditerranée, $\mathrm{n}^{\circ} 18,1974$, p. 68.

7. Kateb Yacine, Nedjma, op. cit., p. 99.

8. Pierre Bourdieu, Sociologie de l'Algérie, Paris, PUF, 1958, p. 15.

9. Kateb Yacine, "La Rose de Blida", in Lettres françaises, n 964, 1963, p. 5.

10. Kateb Yacine, "Vingt ans après", in Révolution africaine, $\mathrm{n}^{\circ}$ 119, 1965, p. 21.

11. Ibid,p. 182.

12. Mohammed Dib, La Grande Maison, Paris, Seuil, 1952.

13. Mohammed Dib, L'Incendie, Paris, Seuil, 1954.

14. Catherine Milkovitch-Rioux, "Figures de la migration chez Kateb Yacine et dans Nedjma", Journée d'études Kateb Yacine, Nedjma, 2009. www.fabula.org/colloques/document1161.php, consulté le 6 août 2011.

15. Kateb Yacine, Le Polygone étoilé, op. cit., p. 11.

16. Jeanne-Marie Clerc, Assia Djebar. Écrire, transgresser, résister, Paris/Montréal, L'Harmattan, 1997, p. 87.

17. Kateb Yacine, "Le rôle d'un écrivain dans un État socialiste", in Dialogues, n 6, 1963, p. 33.

18. Ismail Abdoun, Lectures de Kateb Yacine, Alger, Casbah éditions, 2006, p. 135.

19. Catherine Milkovitch-Rioux, art.cit.

20. Ibid.

21. Ibid.

22. Ibid.

23. Pour notre argumentation, nous retenons ici Nedjma, La Grande Maison et La Soif,écrits en France entre 1948 et 1952, car ils sont tous trois les premiers romans respectifs de Kateb, de Dib et de Djebar.

24. Notons que cela n'est pas propre à Kateb Yacine car nous la retrouvons aussi, au sujet d'autres thématiques, dans la pratique littéraire de Jean Amrouche et de Mohamed Chérif Sahli, par exemple. Comme le note très justement Ismail Abdoun dans ses Lectures de Kateb Yacine, op. cit.: "Cela est lié à l'agression culturelle coloniale. Le recours aux valeurs ancestrales fut, dès le début de la pénétration française, une riposte au mensonge colonial d'inculture des colonisés et un moyen de défense contre l'entreprise de dépersonnalisation."

25. Kateb Yacine, "Le rapport à la langue française", in L'Orient, septembre 1985.

26. Jeanne-Marie Clerc, Assia Djebar. Écrire, résister, transgresser, Paris, L'Harmattan, 1997, p. 33.

27. Kateb Yacine, Le Polygone étoilé, op. cit., p. 9. 